\title{
Staphylococcal Scalded Skin Syndrome in a Breastfed Newborn: A Case Report
}

\author{
Maria Cristina Granado ${ }^{\mathrm{a}, \mathrm{e}} \mathbb{D}$, Ana Lia Goncalo ${ }^{\mathrm{b}}$, Catarina Macedo Francisco ${ }^{\mathrm{c}}$, \\ Sonia Santos ${ }^{\mathrm{c}}$, Joana Verdelho Andrade ${ }^{\mathrm{d}}$, Leonor Carvalho ${ }^{\mathrm{d}}$
}

\begin{abstract}
The authors reveal a clinical report of a 9-day-old newborn girl admitted to the emergency department with generalized erythematous blisters and desquamation without systemic symptoms. A purulent drainage was observed in the left groin area. Staphylococcal scalded skin syndrome (SSSS) was suspected and the newborn was transferred to a pediatric intensive care unit where she started intravenous antibiotics and supportive care. During her stay in the intensive care unit, Staphylococcus aureus (SA) was isolated from the culture obtained from the left groin lesion. A culture from the mother's milk, that did not show any signs of mastitis, was performed which yielded a pure growth of SA. The child improved after empirical treatment with antibiotic. SSSS is potentially life-threatening and thus its early recognition and prompt treatment are crucial to improve patients' outcomes. Further studies are required to better understand the role of breast milk in the transmission of SA.
\end{abstract}

Keywords: Staphylococcus aureus; Scalded skin syndrome; Newborn

\section{Introduction}

Staphylococcal scalded skin syndrome (SSSS), also known as Ritter disease, is a rare blistering skin condition and a potentially life-threatening disorder, particularly in neonates [1-4].

SSSS typically starts with a localized infection in which exfoliative toxins, through hematogenous dissemination,

Manuscript submitted May 10, 2021, accepted June 21, 2021

Published online September 30, 2021

aDepartment of Paediatrics, Hospital Senhora da Oliveira - Guimaraes, Guimaraes, Portugal

${ }^{b}$ Department of Paediatrics, Hospital Pedro Hispano, Porto, Portugal

'Department of Paediatrics, Hospital Sousa Martins, Unidade Local de Saude da Guarda, Guarda, Portugal

dPaediatric Intensive Care Unit, Hospital Pediatrico de Coimbra, Centro Hospitalar Universitario de Coimbra, Coimbra, Portugal

${ }^{e}$ Corresponding Author: Maria Cristina Granado, Department of Paediatrics, Hospital Senhora da Oliveira - Guimaraes, Rua dos Cutileiros, Creixomil, 4835-044 Guimaraes, Portugal. Email: mcristinagranado@gmail.com

doi: https://doi.org/10.14740/ijcp438 cause epidermal damage at distant sites $[1,2,5]$. Clinical findings include skin pain, cutaneous erythema, superficial blistering, desquamation, and constitutional symptoms [5]. The diagnosis is usually clinical and supported by the presence of Staphylococcus aureus (SA) in the cultural exams $[1,2,5]$. Early diagnosis and prompt treatment are crucial to improve patients' prognosis [2-5].

In this particular case, the source of infection appears to be the mother's milk itself as the mother did not present any signs of mastitis. As SA is one of the most frequently isolated bacteria from breast milk, there have been some speculations concerning the routes of SA transmission to infants, namely, if it can be transmitted by their lactating healthy mothers even without mastitis [6-8]. Although extremely rare, neonatal cases of SSSS linked to the presence of SA in the breast milk have been reported [8].

\section{Case Report}

A previously healthy 9-day-old girl, exclusively breastfed, was admitted to the pediatric emergency department with a 3-day history of generalized erythematous blisters and skin erosions without systemic symptoms.

The medical history revealed that the pregnancy and delivery were unremarkable. Serologic test did not reveal active infection during pregnancy, namely, for HIV, syphilis, hepatitis B, rubella, or toxoplasmosis. All prenatal ultrasounds, performed on the first, second and third trimester, were normal. The newborn was a full-term baby, born at 39 weeks of gestational age, vaginal delivery. Anthropometric measurements at birth were: weight $2.905 \mathrm{~kg}$, size $45.5 \mathrm{~cm}$, and head circumference $33 \mathrm{~cm}$. Apgar score was 9 at the first minute and 10 at the fifth minute. The newborn was discharged home on the third day of life with both parents, and she had no siblings. The parents lived in an urban area with adequate sanitation care and good hygiene practices.

The lesions started as a small erythematous blister in the left groin that quickly spread to other parts of the body. Clinical examination on admission (Fig. 1) revealed a widespread tender erythroderma with skin erosions. The lesions were located in the peri-oral region, abdomen, perineal area and both arms and legs. The lesions were more pronounced in the left groin area where a purulent drainage was observed. Nikolsky's sign was positive. Mucosal membranes were spared. Neurological examination revealed irritability, but the remain- 


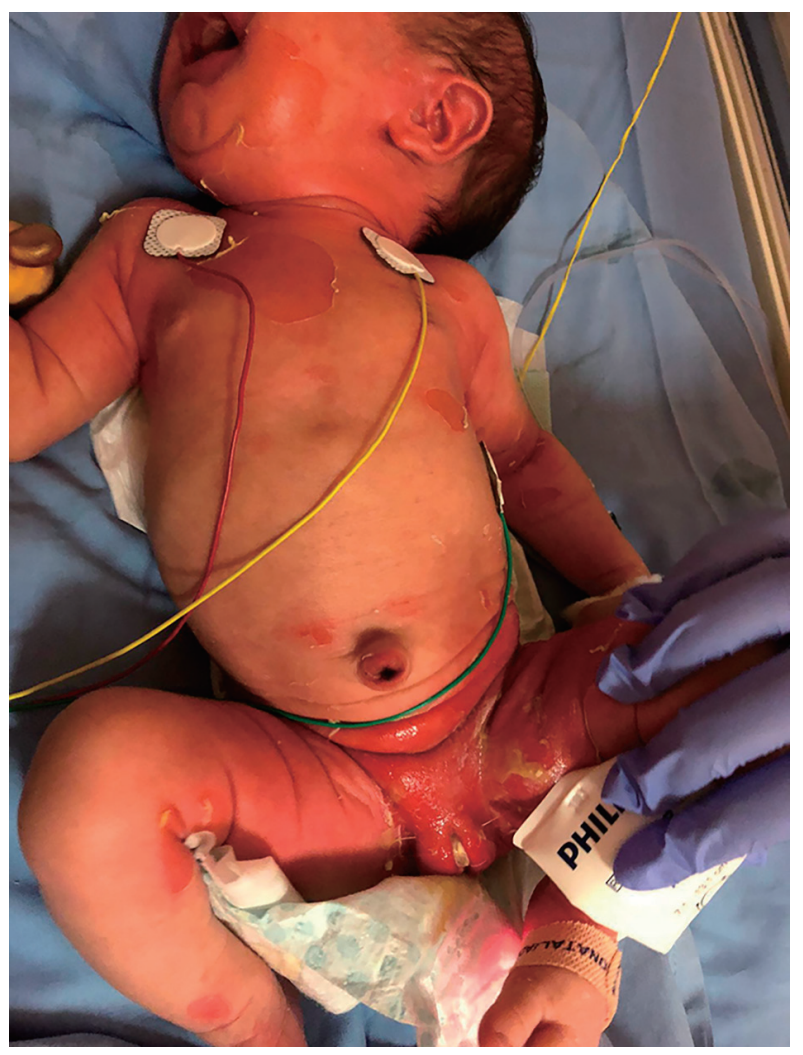

Figure 1. Diffuse erythema and skin erosions in a newborn with staphylococcal scalded skin syndrome. A purulent drainage in the left groin can be observed.

ing neurological exam was unremarkable, namely the level of consciousness, muscle tone and reflexes. Clinical examination revealed no fever with $36.2{ }^{\circ} \mathrm{C}$ of body temperature, the respiratory frequency was $30 \mathrm{cycles} / \mathrm{min}$, the heart rate was 150 beats/min, and the blood pressure was 78/50 (57) $\mathrm{mm} \mathrm{Hg}$. Other body system examinations were normal. The complete blood count revealed hemoglobin of $17.5 \mathrm{~g} / \mathrm{dL}$, leukocytes of $20,620 / \mu \mathrm{L}$ (47\% neutrophils), and platelets of $355,000 / \mu \mathrm{L}$. Liver enzymes, alanine transaminase and aspartate transaminase were normal with a value of $35 \mathrm{U} / \mathrm{L}$ both. Renal function was also preserved with creatinine level of $0.47 \mathrm{mg} / \mathrm{dL}$ and urea nitrogen of $10 \mathrm{mg} / \mathrm{dL}$. C-reactive protein was $0.12 \mathrm{mg} /$ dL. SSSS was suspected. Due to the young age and generalized involvement of the skin, the newborn was transferred to a pediatric intensive care unit. Intravenous antibiotic treatment with flucloxacillin $35 \mathrm{mg} / \mathrm{kg} /$ dose, every $8 \mathrm{~h}$, and clindamycin $5 \mathrm{mg} / \mathrm{kg} /$ dose, every $8 \mathrm{~h}$, was initiated. Topic mupirocin was also initiated and applied in the left groin area twice daily. Supportive measures included intravenous hydration and paracetamol and morphine for pain management, if needed, $15 \mathrm{mg} / \mathrm{kg} /$ dose, every $8 \mathrm{~h}$, and $0.07 \mathrm{mg} / \mathrm{kg} /$ dose, every $4 \mathrm{~h}$, respectively. Within $24 \mathrm{~h}$ of admission, the newborn developed low-grade fever that lasted for 2 days. In the first days of inpatient treatment, a nasogastric tube had to be placed for nourishment. Her vital function remained normal during hospitalization.

A culture was obtained from the left groin lesion. The

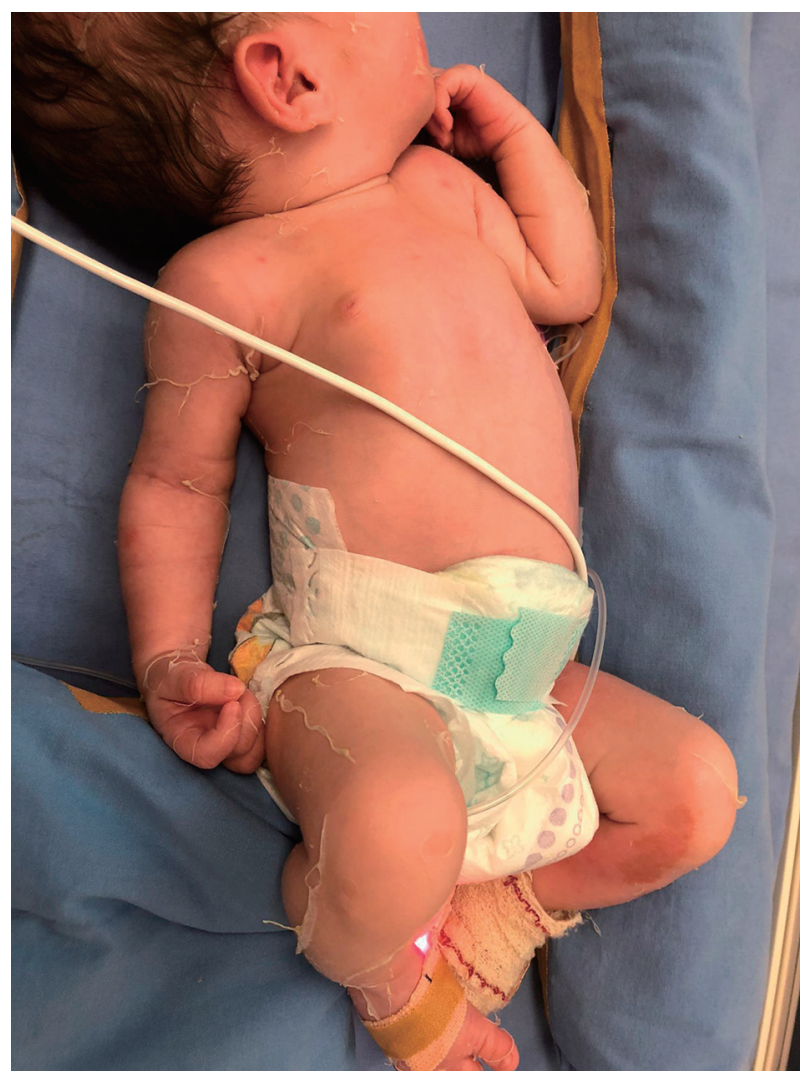

Figure 2. Almost complete skin healing after 8 days of inpatient treatment.

grain stain from the culture obtained from this lesion showed some leukocytes and gram-positive cocci, a penicillin-resistant but methicillin-sensitive SA was isolated. Blood culture was sterile. A culture from the mother's milk was obtained which yielded a pure growth of SA, with the same sensitiveness as the one isolated from the patient's groin lesion. At this point, the mother also started antibiotic therapy and breast milk was interrupted.

Within 3 - 5 days of antibiotic therapy, the erythroderma was decreasing, and desquamation occurred. After 8 days of therapy, the skin was recovering well without any new lesions or scars (Fig. 2). An infant formula was administered, instead of breast milk, in the first 5 days of mother's antibiotic treatment, after that breast milk was re-initiated. Clindamycin was administered for 5 days, and topic mupirocin applied for 7 days. The patient was discharged, on the ninth day of treatment, to the residence area hospital to complete 10 days of intravenous flucloxacillin. This child evolution was favorable, and no complications occurred. At this moment, with 9 months old, she is a healthy child, without other infectious intercurrences, and with a normal psychomotor development.

\section{Discussion}

This case describes an SSSS in a previously healthy neonate that presented to the emergency department with the classical 
clinical features of this syndrome, namely progressive cutaneous erythema, bulla, and desquamation associated to constitutional symptoms. After the beginning of appropriate treatment, the skin lesions started to heal without scarring and the skin recovered completely. No sequels were observed. In this case, an SA was isolated from a left groin lesion of the newborn but also from the mothers' milk. This makes this case interesting as according to the literature only two cases of SSSS were linked to the presence of SA in the breast milk.

SSSS is more frequent in neonates and children younger than 6 years old $[1,2,5]$. These patients are mainly affected probably due to their decreased immunity, lacking toxin-neutralized antibodies, and to their immature renal function with decreased toxin clearance $[1-3,5]$. SSSS is more frequent during summer and autumn months $[1,2,5]$ which is according to our case that occurred during August.

SSSS arises from a focus of infection, usually the nasopharynx, umbilicus or perineum in neonates and young children $[1,2]$. It is caused by two exfoliative toxins produced by certain strains of SA [1-5]. These toxins, exfoliative toxin $\mathrm{A}$ and $\mathrm{B}$, serve as serine proteases enzymes that target and cleave desmoglein 1, a desmosomal-linking protein responsible for keratinocytes adhesion in the superficial epidermis [2, 5]. Once released in the bloodstream, the exfoliative toxins reach the superficial epidermis, where they act locally, causing splitting of the desmosomes which results in the characteristic skin lesion of this syndrome: erythema, bulla formation and desquamation [2-5]. Desmoglein 1 is found in the upper epidermis but not in mucosal membranes which explains the absence of mucosal membrane involvement in SSSS [2].

Transmission of exotoxin-producing SA strains can occur between individuals, including asymptomatic colonized individuals, by direct contact $[5,6]$. Outbreaks of SSSS among neonates usually occur secondarily to parents or health care workers being asymptomatic carriers of these strains [1]. As SA is transmitted by direct contact, there have been some speculations concerning the routes of its transmission in infants colonized with this agent [6]. SA is the most frequently bacteria associated with infectious mastitis [6] and it can be the source of infection for breastfed newborns [5]. SA is also one of the most frequently isolated pathogens in breast milk [7] and it is suggested that it can be transmitted by lactating healthy mothers without mastitis $[6,8]$. According to Fertitta et al [8], only two neonatal cases of SSSS were linked to the presence of SA in the breast milk. In our case, the mother did not present any symptoms or signs of mastitis but her milk culture yield a pure growth of SA. This finding leads us to question if the mother's breast milk was the source of the infection to the newborn.

SSSS starts with skin tender erythema, initially accentuated in skin folds and periorificial areas, that quickly spreads [1-5]. As the disease progresses, blisters develop in the erythematous areas and enlarge to form bullae that easily rupture [1, 2]. Superficial desquamation occurs leaving extensive areas of denuded skin with scalded appearance $[2,5]$. Nikolsky sign is positive and elicited by applying gentle pressure on skin, resulting in extension of the blisters and separation of the epidermis of the adjacent skin $[1,2]$. Mucosal involvement is absent $[1,2,5]$. Skin pain, malaise, irritability, poor feeding, and fever can be present $[1,2,5]$. The source of infection is not often evident [5]. Findings suggestive of its location include erosions, purulent drainage or crusting areas on the skin [5].

The differential diagnosis includes other skin conditions that present with extensive erythema or blistering namely burns, bullous impetigo, epidermolysis bullosa, StevensJohnson syndrome, toxic epidermal necrolysis, toxic shock syndrome, Kawasaki disease, and pemphigus [2, 3, 5].

The diagnosis of SSSS is usually clinical but bacterial cultures of suspected primary focus of infection or, in its absence, from a common site of SA colonization, should be performed to confirm the diagnosis and provide information on antibiotic susceptibility $[1,2,5]$. Culturing exfoliative lesions and bullae is not helpful because these lesions result from hematologic dissemination of SA exotoxins rather than local infection $[1,2,5]$. Therefore, cultures should be taken from cutaneous sites of suspected primary infection or, in its absence, from a common site of SA colonization, namely the nasopharynx, axillae, umbilicus or perineum $[2,5]$. Blood cultures are usually negative in otherwise healthy children $[2,5]$.

The management of SSSS involves eradication of SA and supportive care $[1,5]$. Inpatient admission is required to decrease morbidity and mortality $[3,5]$. Prompt empiric treatment with an antistaphylococcal penicillins intravenous antibiotic, such as flucloxacillin, is crucial until cultures are available to guide therapy $[1,2,5]$. If the patient is critically ill, not improving or in communities where the prevalence of methicillin-resistant SA is high, vancomycin should be started $[1,2]$. Clindamycin can be added because of its capacity to reduce bacterial toxin production $[1,2]$. Topical antibiotics, such as fusidic acid and mupirocin, can be used as adjuvant therapy in an attempt to eradicate colonization [1-3]. Antibiotic therapy should be continued for at least 10 days [1, 5] and extended to 14 days in patients with a slower response to treatment [5].

Supportive care includes wound care with minimal manipulation, maintenance of fluid and electrolyte balance, nutritional support, and pain and fever management [2-5].

Complications of SSSS include secondary infections, hypothermia, dehydration/electrolyte imbalance, and death [2, $3,5]$.

Unless complications develop, the prognosis is mostly favorable [3]. Even with extensive lesions scarring is rare and healing occurs within 2 - 3 weeks [2-5]. In pediatric patients, the mortality rate is about $3-5 \%$ and mostly associates with complications $[1,2,4]$. Recurrence is rare [5].

\section{Learning points}

Although rare, SSSS is a potentially life-threatening disorder, and thus a high index of suspicion is necessary to improve the patients' outcome. Diagnosis is usually based on characteristic clinical findings: erythema, bulla and desquamation. Appropriate antimicrobial therapy with an antistaphylococcal penicillin typically results in rapid clinical improvement without sequels. Further investigation is required to better understand the role of breast milk in SA transmission to breastfed infants. 


\section{Acknowledgments}

None to declare.

\section{Financial Disclosure}

None to declare.

\section{Conflict of Interest}

None to declare.

\section{Informed Consent}

Informed consent was obtained.

\section{Author Contributions}

MCG, LG, CMF, SS, JA and LC participated in the management of the patient and gave substantial contributions to this work. MCG wrote the manuscript with support from LG and CMF. Review and editing: SS, JA and LC. Each author has given final approval of the final version.

\section{Data Availability}

The authors declare that data supporting the findings of this study are available within the article.

\section{Abbreviations}

SSSS: staphylococcal scalded skin syndrome; SA: Staphylococcus aureus

\section{References}

1. Dollani LC, Marathe KS. Impetigo/staphylococcal scalded skin disease. Pediatr Rev. 2020;41(4):210-212.

2. Leung AKC, Barankin B, Leong KF. Staphylococcalscalded skin syndrome: evaluation, diagnosis, and management. World J Pediatr. 2018;14(2):116-120.

3. Jordan KS. Staphylococcal scalded skin syndrome: a pediatric dermatological emergency. Adv Emerg Nurs J. 2019;41(2):129-134.

4. Kouakou K, Dainguy ME, Kassi K. Staphylococcal scalded skin syndrome in neonate. Case Rep Dermatol Med. 2015;2015:901968.

5. McMahon P. Staphylococcal scalded skin syndrome. [Accessed September 6, 2020]. Available at: https://uptodate. com.

6. Kawada M, Okuzumi K, Hitomi S, Sugishita C. Transmission of Staphylococcus aureus between healthy, lactating mothers and their infants by breastfeeding. J Hum Lact. 2003;19(4):411-417.

7. Li X, Zhou Y, Zhan X, Huang W, Wang X. Breast milk is a potential reservoir for livestock-associated staphylococcus aureus and community-associated staphylococcus aureus in Shanghai, China. Front Microbiol. 2017;8:2639.

8. Fertitta L, Welfringer-Morin A, Rigourd V, Jamet A, Hadj-Rabia S, Lesage F, Bodemer C. Neonatal staphylococcal scalded skin syndrome in a breastfed neonate. J Eur Acad Dermatol Venereol. 2020;34(1):e36-e38. 